\title{
A new approach on the modelling, chaos control and synchronization of a fractional biological oscillator
}

\author{
Ali Saleh Alshomrani ${ }^{*}$, Malik Zaka Ullah¹ and Dumitru Baleanu ${ }^{2,3}$
}

\section{"Correspondence:}

aszalshomrani@kau.edu.sa

'Mathematical Modelling and

Applied Computation Research

Group (MMAC), Department of

Mathematics, Faculty of Science,

King Abdulaziz University, Jeddah

21589, Saudi Arabia

Full list of author information is

available at the end of the article

\section{Springer}

\begin{abstract}
This research aims to discuss and control the chaotic behaviour of an autonomous fractional biological oscillator. Indeed, the concept of fractional calculus is used to include memory in the modelling formulation. In addition, we take into account a new auxiliary parameter in order to keep away from dimensional mismatching. Further, we explore the chaotic attractors of the considered model through its corresponding phase-portraits. Additionally, the stability and equilibrium point of the system are studied and investigated. Next, we design a feedback control scheme for the purpose of chaos control and stabilization. Afterwards, we introduce an efficient active control method to achieve synchronization between two chaotic fractional biological oscillators. The efficiency of the proposed stabilizing and synchronizing controllers is verified via theoretical analysis as well as simulations and numerical experiments.
\end{abstract}

Keywords: Fractional model; Biological system; Chaotic attractors; Chaos control; Synchronization

\section{Introduction}

Chaos is one of the most prominent features of complex dynamical systems whose state variables are highly dependent on their initial conditions. This property may lead to the divergence of nonlinear chaotic systems; therefore, the analysis of such phenomena is of great importance from both theoretical and practical points of view [1].

Because of the wide appearance of chaotic systems in different fields of study such as secure communications, physics, biology and economy, a growing interest has been devoted to the control and synchronization of chaotic processes [2]. In [3], a new systematic approach within the use of scalar transmitted signal was designed to synchronize a class of hyperchaotic systems. In [4], a synchronization approach was presented for chaotic/hyperchaotic systems by formulating a linear feedback control problem. In [5], two identical hyperchaotic systems were synchronized by using a nonlinear control technique. In [6], a parameter observer was designed in order to identify unknown parameters in hyperchaotic systems, which was needed for designing a state-feedback controller. The author in [7] controlled and synchronized chaotic systems using state-dependent

(c) The Author(s) 2021. This article is licensed under a Creative Commons Attribution 4.0 International License, which permits use, sharing, adaptation, distribution and reproduction in any medium or format, as long as you give appropriate credit to the original author(s) and the source, provide a link to the Creative Commons licence, and indicate if changes were made. The images or other third party material in this article are included in the article's Creative Commons licence, unless indicated otherwise in a credit line to the material. If material is not included in the article's Creative Commons licence and your intended use is not permitted by statutory regulation or exceeds the permitted use, you will need to obtain permission directly from the copyright holder. To view a copy of this licence, visit http://creativecommons.org/licenses/by/4.0/. 
Riccati equations. In [8], a newly introduced fractional-order hyperchaotic system was controlled and synchronized by choosing an appropriate Lyapunov function and using a state-feedback control computation. In [9], Lorenz and Chen systems were considered in the presence of parameter uncertainty and controlled by using an adaptive dual synchronization controller. In [10], an adaptive terminal sliding mode control was applied to the chaotic Chen and hyperchaotic Lorenz systems. In [11], considering the stability theory of Lyapunov, the authors synchronized three nonidentical systems of different dimensions.

Recently, the investigation of chaos in biology has attracted the attention of many scientists due to the unpredictable behaviour of biological processes. In [12], the authors controlled the chaotic behaviour of tumor cells using a non-feedback loop. In [13], the author designed an optimal drug delivery schedule for the chaotic behaviour of tumor cells by minimizing Hamiltonian function; then he controlled the same system considering uncertainty in the chaotic model. In [14], the author analysed the chaotic behaviour of Lotka-Voltera biological systems. In [15], the authors synchronized two fractional chaotic maps by using a feedback control method. In [16], a fuzzy adaptive controller was designed to achieve a projective synchronization for a class of fractional chaotic systems with input nonlinearities.

Nowadays, a noticeable number of sensational researches have considered fractionalorder systems due to their memory-oriented features, a fact which makes such systems more realistic to describe real-world dynamics [17-23]. Thus, it is very practical to investigate and analyse chaos in fractional-order systems in order to simulate the complex behaviour of realistic phenomena. In addition, efficient stabilization and synchronization techniques are needed to be developed in order to overcome the chaotic behaviour of such fractional processes. Thus, the problem of chaos control and synchronization for complex fractional-order systems has been of great importance to a wide audience. More to the point, efficient numerical methods should be extended to deal with controversial problems in fractional calculus like modelling and control [24-26]. Inspired by the aforementioned argument, this paper introduces a new fractional model for a chaotic biological oscillator. We study the stability and equilibrium of the new system and analyse its chaotic behaviour by using phase-portrait responses. Then we propose a state-feedback control to stabilize the fractional model and apply an active control strategy to synchronize two identical chaotic oscillators. The applicability of the proposed controllers is also verified through theoretical analysis as well as some simulation results.

The reminder of this paper is organized as follows. In Sect. 2, some preliminary results are presented. In Sect. 3, the new mathematical fractional model of a chaotic biological oscillator is introduced, and its stability and equilibrium point are investigated. Afterwards, a state-feedback control is designed to stabilize the new model, and an active controller is introduced in order to achieve synchronization. Finally, the paper is closed by some concluding remarks in the last section.

\section{Preliminaries}

In this section, we recall the basic definition of Caputo fractional derivative and introduce its corresponding integral operator. The Laplace transform and the antiderivative property of this definition are also presented in the sequel. 
Definition 2.1 ([27]) Let $q \in(0,1)$ and $f$ be an integrable function. Then the $q$ th-order Caputo fractional derivative of $f$ is defined by

$$
{ }^{C} \mathscr{D}^{q} f(t)=\frac{1}{\Gamma(1-q)} \int_{0}^{t} \dot{f}(\tau)(t-\tau)^{-q} d \tau .
$$

The associated $q$ th-order Riemann-Liouville fractional integral of $f$ is also defined as

$$
\mathscr{I}^{q} f(t)=\frac{1}{\Gamma(q)} \int_{0}^{t} f(\tau)(t-\tau)^{q-1} d \tau
$$

whenever the integral exists.

For the $q$ th-order Caputo derivative when $q \in(0,1)$, the Laplace transform is introduced by

$$
\mathscr{L}\left[{ }^{C} \mathscr{D}^{q} f(t)\right]=s^{q} F(s)-f(0),
$$

where $F(s)=\mathscr{L}[f(t)]$. In addition, the fractional derivative (1), together with the integral operator (2), satisfies the antiderivative property such that

$$
\mathscr{I}^{q}\left[{ }^{C} \mathscr{D}^{q} f(t)\right]=f(t)-f(0) .
$$

\section{The new fractional model}

In the recent study [28], the authors employed an autonomous model to describe the interaction between enzyme and substrate mechanism. In [28], the aforesaid model was introduced in the form of the following system of ordinary differential equations:

$$
\left\{\begin{array}{l}
\dot{f}_{1}=f_{2}, \\
\dot{f}_{2}=\alpha f_{3}, \\
\dot{f}_{3}=f_{4}, \\
\dot{f}_{4}=-f_{1}-\beta f_{3}+\gamma f_{2}-f_{4}+\varepsilon f_{2}\left(1-f_{1}^{2}+\eta f_{1}^{4}-\kappa f_{1}^{6}\right),
\end{array}\right.
$$

where the coefficients $\alpha, \beta, \gamma, \varepsilon, \eta, \kappa$ are real constants. As mentioned in [28], the model (5) exhibits chaotic attractors when the parameters $(\alpha, \beta, \gamma, \varepsilon, \eta, \kappa)$ take the values $(5,24,-0.05,2.001,2.55,1.7)$, respectively. However, the integer-order model (5) suffers from the lack of memory effect, while hereditary property is the intrinsic feature of many complex biological systems. To overcome this drawback, the concept of fractional calculus is used to include memory in the model formulation. To do so, we extend the model (5) by substituting the integer-order derivatives with fractional Caputo ones defined in (1). Moreover, we take into account the new auxiliary parameter $\theta$ in order to keep away from dimensional mismatching [29]. Thus, we formulate the new fractional biological oscillator by

$$
\left\{\begin{array}{l}
\frac{1}{{ }^{1-q}} C \mathscr{D}^{q} f_{1}=f_{2}, \\
\frac{1}{{ }^{1-q}} C \mathscr{D}^{q} f_{2}=\alpha f_{3}, \\
\frac{1}{{ }^{1-q}} C \mathscr{D}^{q} f_{3}=f_{4}, \\
\frac{1}{\theta^{1-q}} C \mathscr{D}^{q} f_{4}=-f_{1}-\beta f_{3}+\gamma f_{2}-f_{4}+\varepsilon f_{2}\left(1-f_{1}^{2}+\eta f_{1}^{4}-\kappa f_{1}^{6}\right) .
\end{array}\right.
$$


Meanwhile, please note that the model (6) is the extension of a generalized Van der Pol oscillator; hence, this model can describe various biological and bio-physical phenomena.

\subsection{Equilibrium point and stability}

Considering the certain values of parameters $(\alpha, \beta, \gamma, \varepsilon, \eta, \kappa)=(5,24,-0.05,2.001,2.55$, 1.7), we obtain the equilibrium point of the model (6) from

$$
\left\{\begin{array}{l}
f_{2}=0, \\
\alpha f_{3}=0, \\
f_{4}=0, \\
-f_{1}-\beta f_{3}+\gamma f_{2}-f_{4}+\varepsilon f_{2}\left(1-f_{1}^{2}+\eta f_{1}^{4}-\kappa f_{1}^{6}\right)=0 .
\end{array}\right.
$$

Solving the system of algebraic equations (7), we find that the system (6) has a unique equilibrium point $f^{*}=\left(f_{1}^{*}, f_{2}^{*}, f_{3}^{*}, f_{4}^{*}\right)=(0,0,0,0)$. Then we compute the Jacobian matrix at the point $f^{*}$

$$
J^{*}=\left[\begin{array}{cccc}
0 & 1 & 0 & 0 \\
0 & 0 & 5 & 0 \\
0 & 0 & 0 & 1 \\
-1 & 1.951 & -24 & -1
\end{array}\right]
$$

whose eigenvalues are

$$
\lambda_{1,2}=0.2468 \pm 0.4279 i, \quad \lambda_{3,4}=-0.7468 \pm 4.4649 i .
$$

Now, we list two well-known lemmas discussing the stability of fractional-order systems.

Lemma 3.1 ([30]) The equilibrium point $f^{*}$ of the fractional-order system (6) is locally asymptotically stable if the following inequality holds:

$$
\left|\arg \left(\lambda_{i}\right)\right|>\frac{\pi}{2} q, \quad i=1,2,3,4,
$$

where $q$ is the fractional order, and $\lambda_{i}$ is the ith eigenvalue of $J^{*}$ at the point $f^{*}$.

Lemma 3.2 ([31]) Let the characteristic equation of the model (6) at $f^{*}$ be in the form

$$
\operatorname{det}\left(\lambda I-J^{*}\right)=\lambda^{4}+a_{3} \lambda^{3}+a_{2} \lambda^{2}+a_{1} \lambda^{1}+a_{0}=0 .
$$

Then the equilibrium $f^{*}$ is locally asymptotically stable for all $q \in(0,1)$ if the corresponding Routh-Hurwitz table has elements with the same sign in its first column.

\subsection{Simulation results}

In the following, we simulate the complex behaviour of the new modified model (6) for the fractional orders $q=0.9,0.92,0.94,0.96,0.98,1$. To this end, we consider the initial conditions as

$$
f(0)=\left(f_{1}(0), f_{2}(0), f_{3}(0), f_{4}(0)\right)=(0.5,0.5,0.5,0.5),
$$



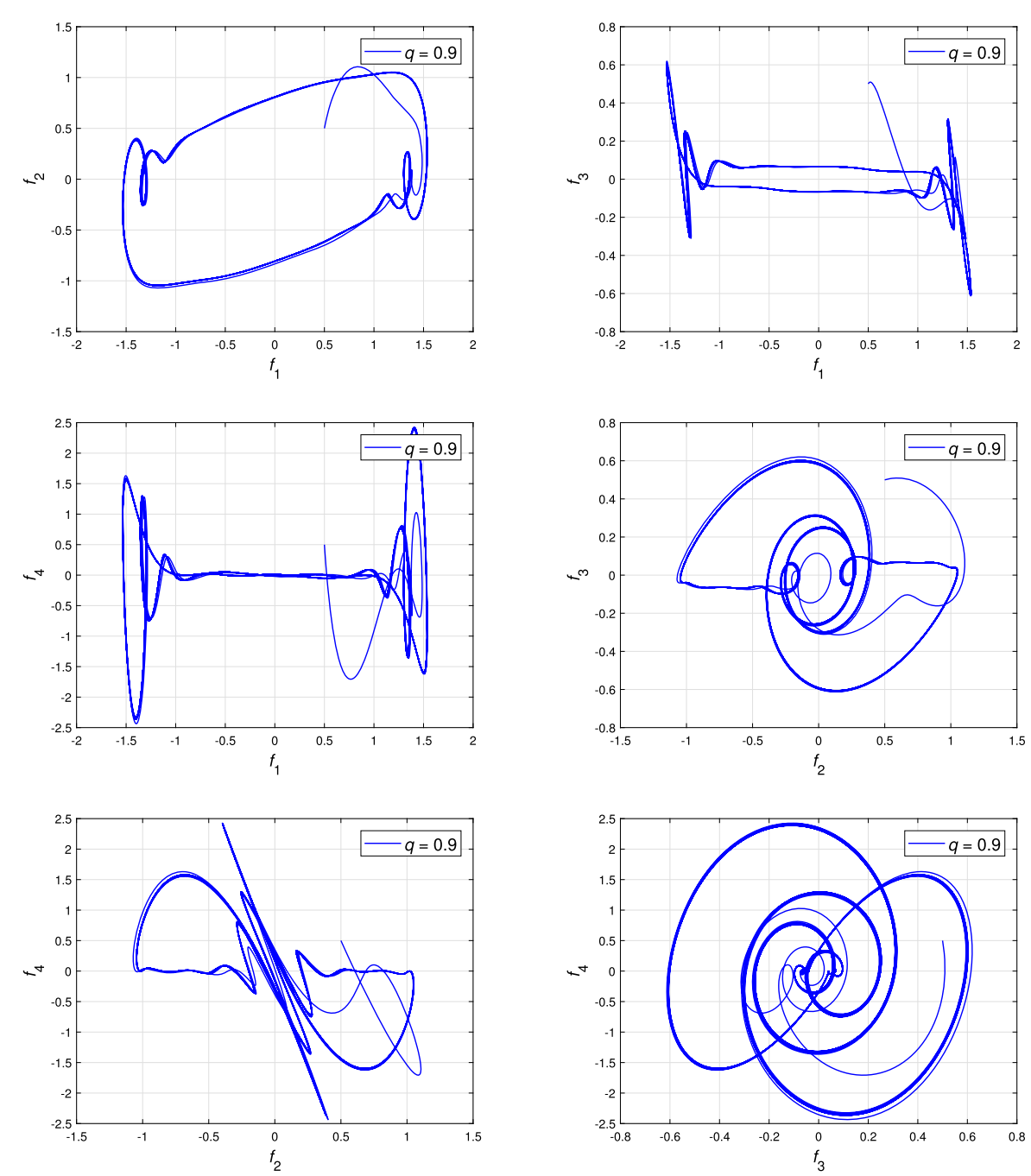

Figure 1 The phase planes of the fractional chaotic system (6) for $q=0.9$

and select the parameters from

$$
(\alpha, \beta, \gamma, \varepsilon, \eta, \kappa, \theta)=(5,24,-0.05,2.001,2.55,1.7,0.99)
$$

Figures 1-6 depict the relevant phase-portraits and verify the existence of complex chaotic attractors for the considered values of the fractional order $q$.

Remark 3.1 Concerning the numerical simulation, here we applied the predictorcorrector method [32] in which an FFT algorithm is employed to evaluate the discrete convolutions [33]. The accuracy and the convergence of this scheme were studied in [34]. Also, contrary to the classical implementation in which the computational cost is proportional to $N^{2}$, here we have $N \log \left(N^{2}\right)$ for the computational cost when the solution is evaluated at $N$ time-points. 

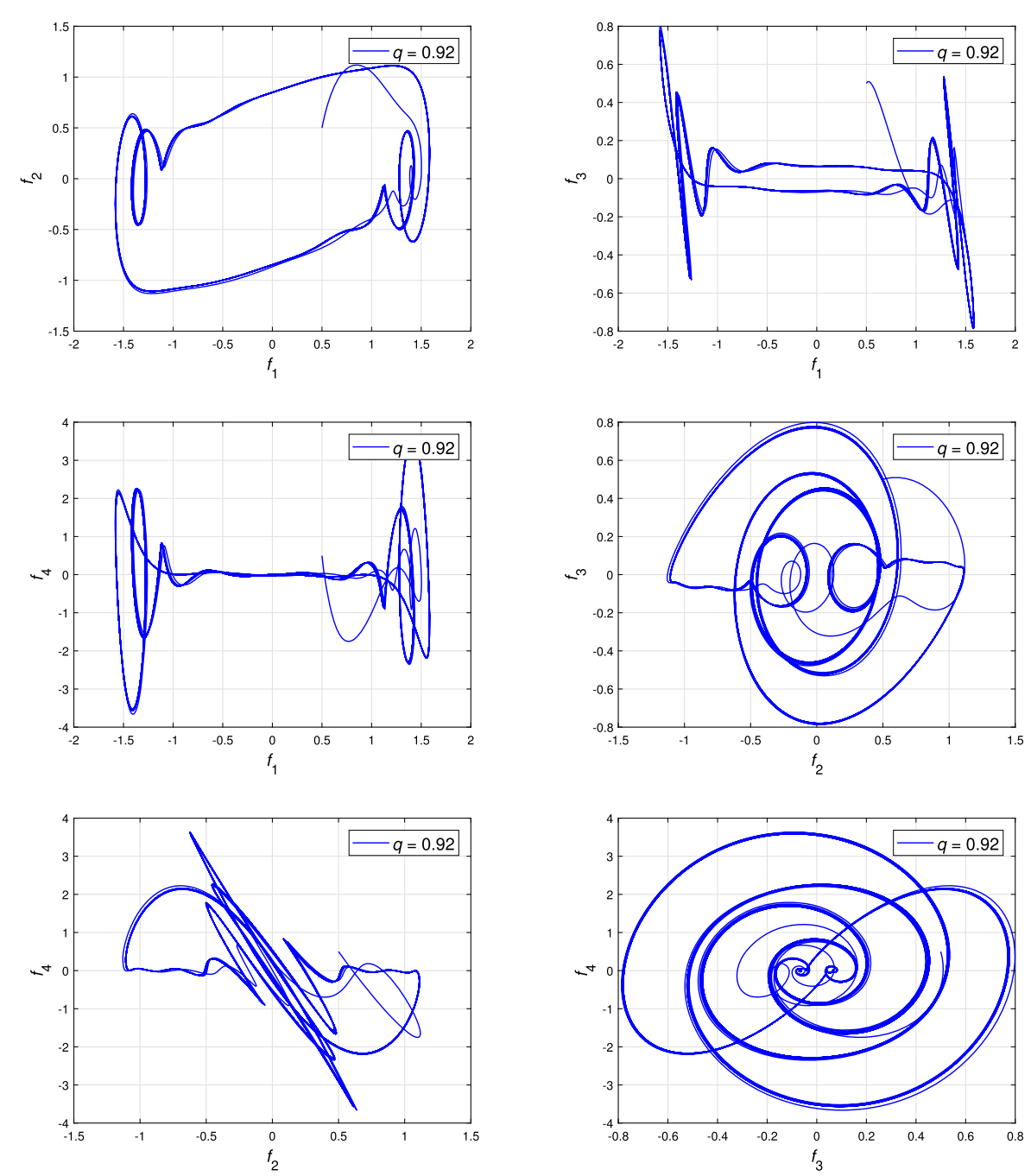

Figure 2 The phase planes of the fractional chaotic system (6) for $q=0.92$

\section{Chaos control and synchronization}

In this section, designing a state-feedback controller, we compensate the undesirable chaotic behaviour of the fractional-order biological system (6). Then we introduce an active control approach to synchronize two identical fractional chaotic oscillators.

\subsection{Chaos control}

In order to control the chaotic behaviour of the fractional-order model (6), we consider the following modified controlled system:

$$
\left\{\begin{array}{l}
\frac{1}{\bar{\theta}^{1-q}} C \mathscr{D}^{q} f_{1}=f_{2}-u_{1}\left(f_{1}-f_{1}^{*}\right), \\
\frac{1}{{ }^{1-q}} C \mathscr{D}^{q} f_{2}=\alpha f_{3}-u_{2}\left(f_{2}-f_{2}^{*}\right), \\
\frac{1}{\bar{\theta}^{1-q}} C \mathscr{D}^{q} f_{3}=f_{4}-u_{3}\left(f_{3}-f_{3}^{*}\right), \\
\frac{1}{\theta^{1-q}} C \mathscr{D}^{q} f_{4}=-f_{1}-\beta f_{3}+\gamma f_{2}-f_{4}+\varepsilon f_{2}\left(1-f_{1}^{2}+\eta f_{1}^{4}-\kappa f_{1}^{6}\right)-u_{4}\left(f_{4}-f_{4}^{*}\right),
\end{array}\right.
$$



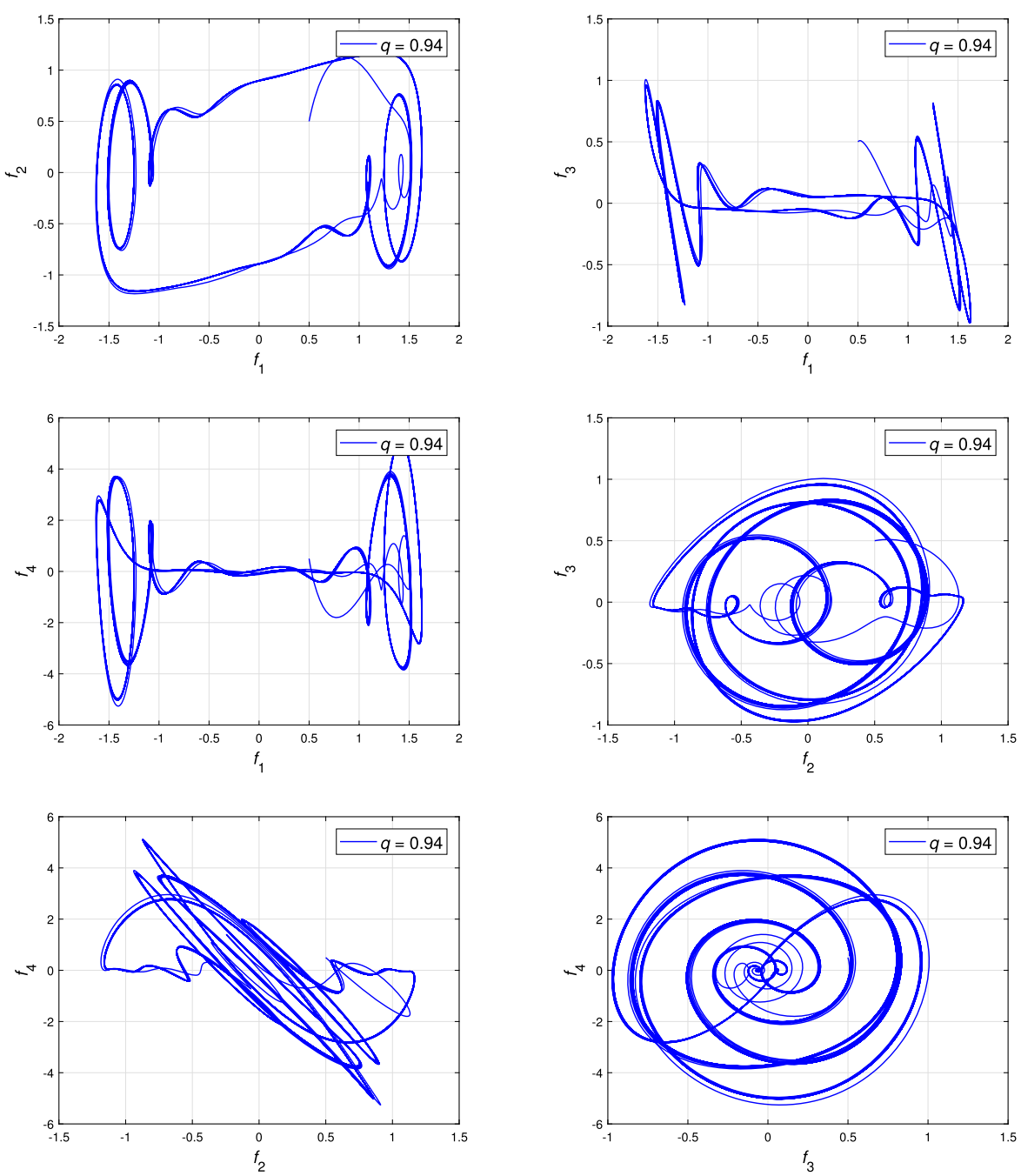

Figure 3 The phase planes of the fractional chaotic system (6) for $q=0.94$

where $u_{i} \geq 0$ is the state-feedback control gain. The objective is to design $u_{i}$ in such a way that the state variables $f_{1}, f_{2}, f_{3}, f_{4}$ of the controlled system (14) converge to the steadystate $f^{*}=(0,0,0,0)$ asymptotically. Considering the system parameters $(\alpha, \beta, \gamma, \varepsilon, \eta, \kappa, \theta)=$ $(5,24,-0.05,2.001,2.55,1.7,0.99)$, we compute the Jacobian matrix $J^{*}$ at the point $f^{*}=$ $\left(f_{1}^{*}, f_{2}^{*}, f_{3}^{*}, f_{4}^{*}\right)=(0,0,0,0)$ as

$$
J^{*}=\left[\begin{array}{cccc}
-u_{1} & 1 & 0 & 0 \\
0 & -u_{2} & 5 & 0 \\
0 & 0 & -u_{3} & 1 \\
-1 & 1.951 & -24 & -u_{4}-1
\end{array}\right] .
$$

Then we derive

$$
\lambda I-J^{*}=\left[\begin{array}{cccc}
\lambda+u_{1} & -1 & 0 & 0 \\
0 & \lambda+u_{2} & -5 & 0 \\
0 & 0 & \lambda+u_{3} & -1 \\
1 & -1.951 & 24 & \lambda+u_{4}+1
\end{array}\right],
$$



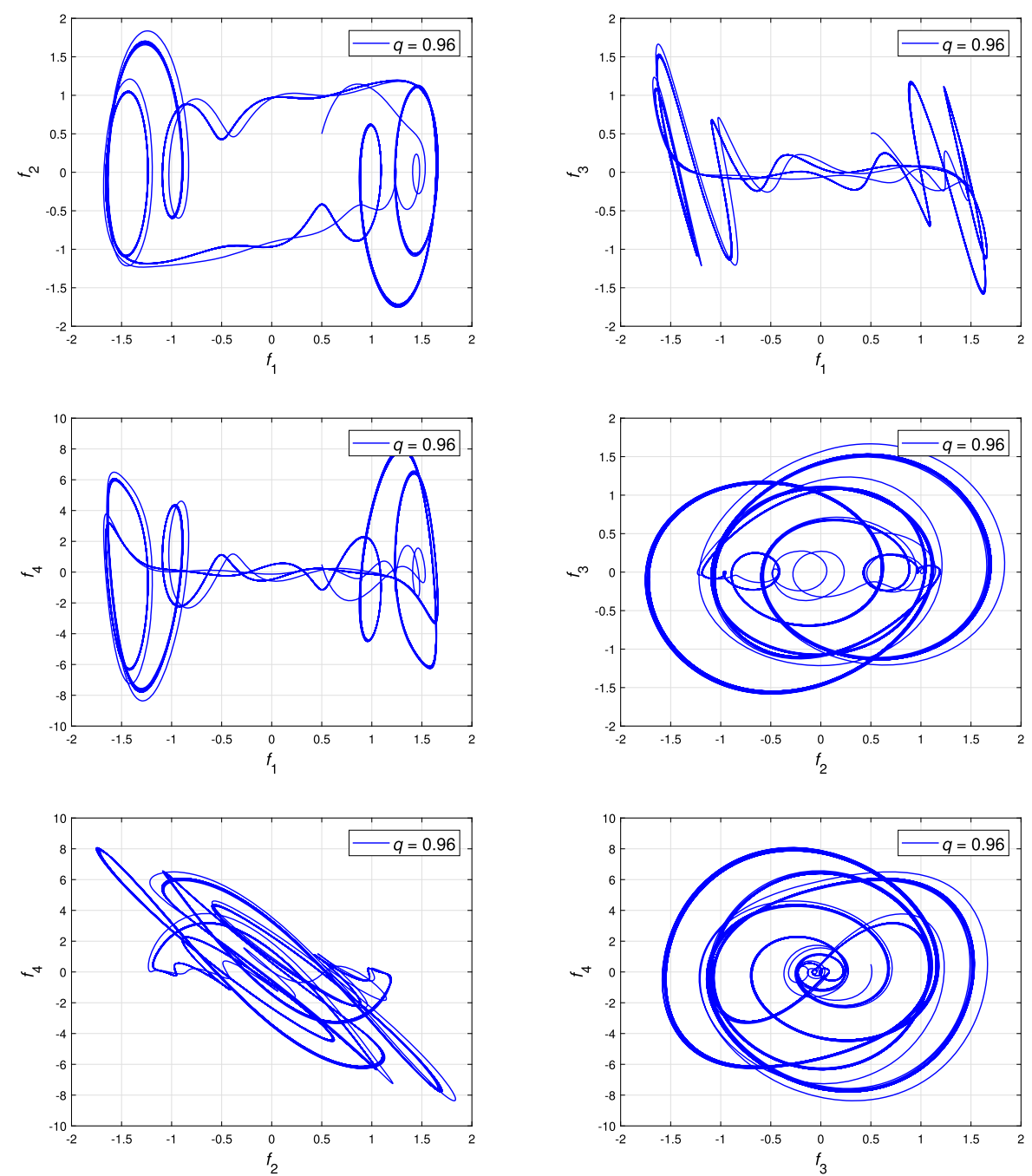

Figure 4 The phase planes of the fractional chaotic system (6) for $q=0.96$

whose determinant is obtained in the form $\operatorname{det}\left(\lambda I-J^{*}\right)=\lambda^{4}+a_{3} \lambda^{3}+a_{2} \lambda^{2}+a_{1} \lambda^{1}+a_{0}=0$ where

$$
\begin{aligned}
a_{3}= & 1+u_{2}+u_{3}+u_{4}+u_{1}, \\
a_{2}= & 24+u_{2}+u_{3}+u_{1}+u_{1} u_{2}+u_{1} u_{3}+u_{3} u_{4}+u_{2} u_{4}+u_{1} u_{4}+u_{2} u_{3}, \\
a_{1}= & -9.755+24 u_{2}+24 u_{1}+u_{2} u_{3} u_{4}+u_{1} u_{2} u_{3}+u_{1} u_{2} u_{4}+u_{1} u_{3} u_{4} \\
& +u_{1} u_{2}+u_{1} u_{3}+u_{2} u_{3},
\end{aligned}
$$$$
a_{0}=5+24 u_{1} u_{2}+u_{1} u_{2} u_{3} u_{4}-9.755 u_{1}+u_{1} u_{2} u_{3} .
$$

For the state-feedback gains $\left(u_{1}, u_{2}, u_{3}, u_{4}\right)=(0.25,0.25,0.25,0.25)$, we obtain

$$
\operatorname{det}\left(\lambda I-J^{*}\right)=\lambda^{4}+2 \lambda^{3}+25.125 \lambda^{2}+2.495 \lambda+4.08078125=0,
$$



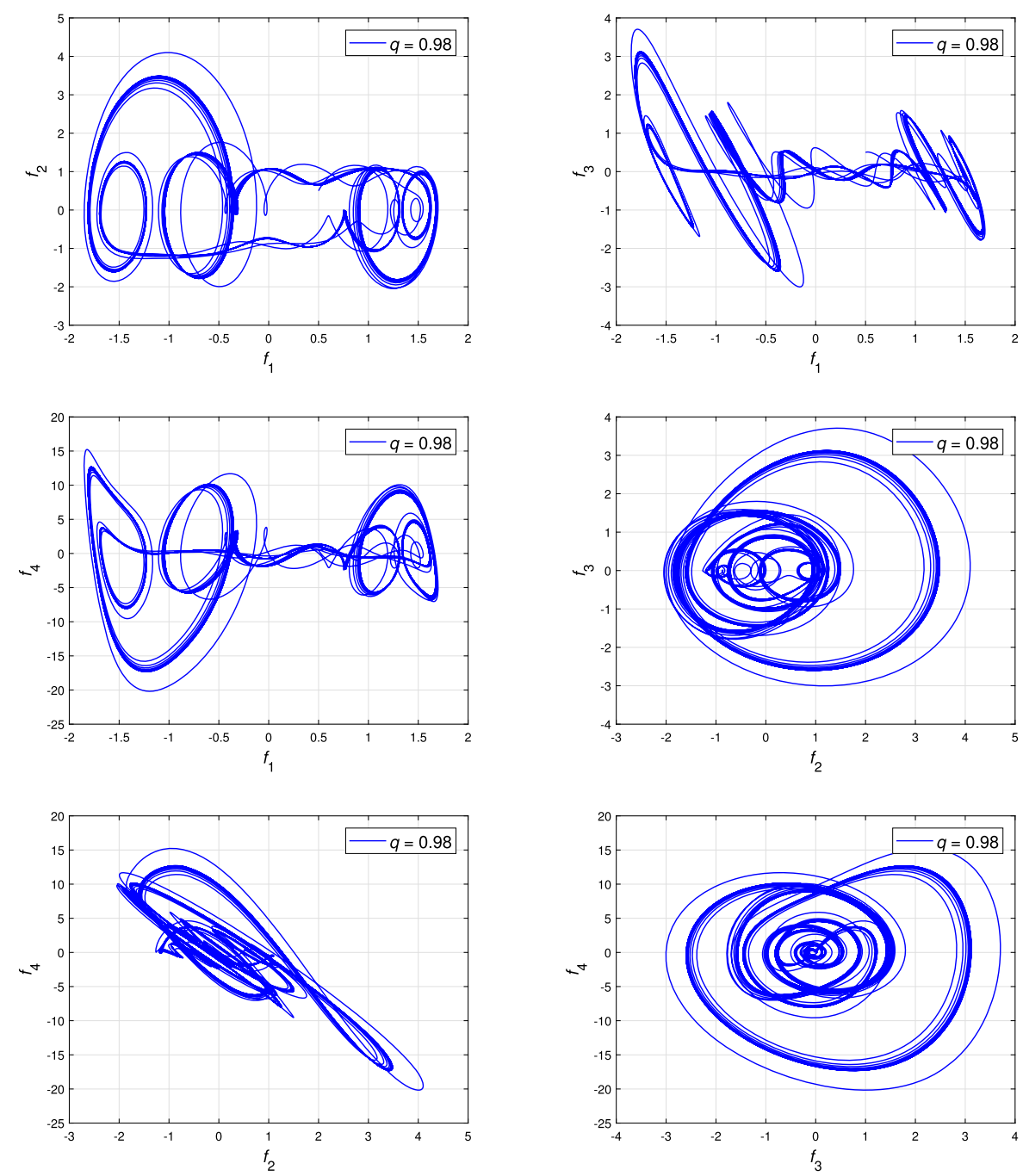

Figure 5 The phase planes of the fractional chaotic system (6) for $q=0.98$

and the corresponding Routh-Hurwitz table of stability is given in Table 1. As can be seen, we get the satisfaction of stability condition in Lemma 3.2, so the equilibrium $f^{*}$ of the controlled system (14) is locally asymptotically stable for all $q \in(0,1)$. We depict the controlled states in Figs. 7-12 for the fractional orders $q=0.9,0.92,0.94,0.96,0.98,1$ and the initial conditions $f(0)=(0.5,0.5,0.5,0.5)$. Simulation results confirm the validity of the proposed approach to overcome the undesirable behaviour of chaotic attractors.

\subsection{Synchronization}

In this section, two identical fractional-order chaotic systems are synchronized by an active control scheme. To do so, the master and the slave systems are, respectively, consid- 

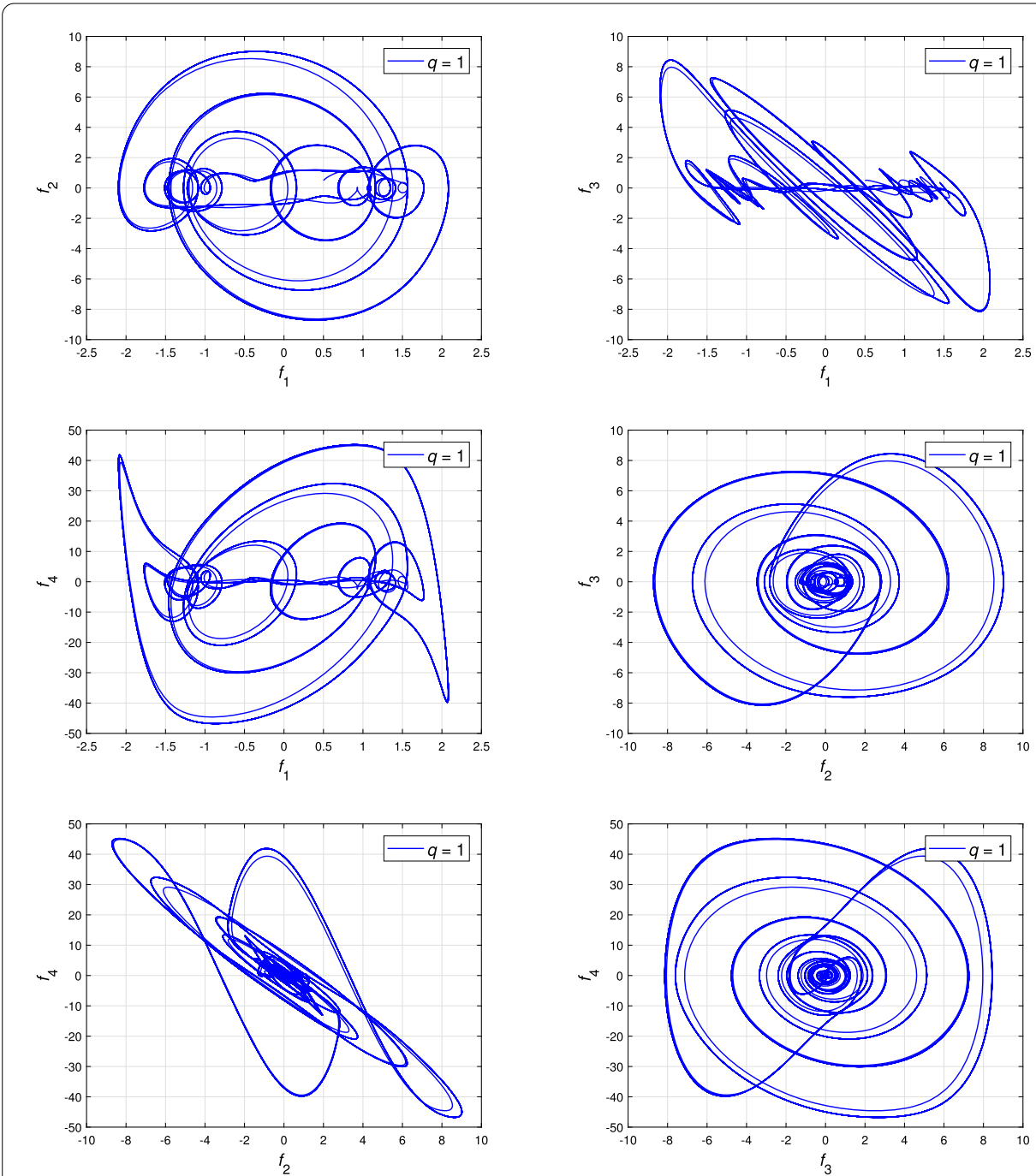

Figure 6 The phase planes of the fractional chaotic system (6) for $q=1$

Table 1 Routh-Hurwitz table of stability

\begin{tabular}{llll}
\hline$\lambda^{4}$ & 1 & 25.12 & 4.08078125 \\
$\lambda^{3}$ & 2 & 2.495 & 0 \\
$\lambda^{2}$ & 23.8725 & 4.08078125 & 0 \\
$\lambda^{1}$ & 2.153118651 & 0 & 0 \\
$\lambda^{0}$ & 4.08078125 & 0 & 0 \\
\hline
\end{tabular}

ered as

$$
\left\{\begin{array}{l}
\frac{1}{\theta^{1-q}} C \mathscr{D}^{q} f_{1}=f_{2}, \\
\frac{1}{\theta^{1-q}} C \mathscr{D}^{q} f_{2}=\alpha f_{3}, \\
\frac{1}{\theta^{1-q}} C \mathscr{D}^{q} f_{3}=f_{4}, \\
\frac{1}{\theta^{1-q}} C \mathscr{D}^{q} f_{4}=-f_{1}-\beta f_{3}+\gamma f_{2}-f_{4}+\varepsilon f_{2}\left(1-f_{1}^{2}+\gamma f_{1}^{4}-\kappa f_{1}^{6}\right),
\end{array}\right.
$$



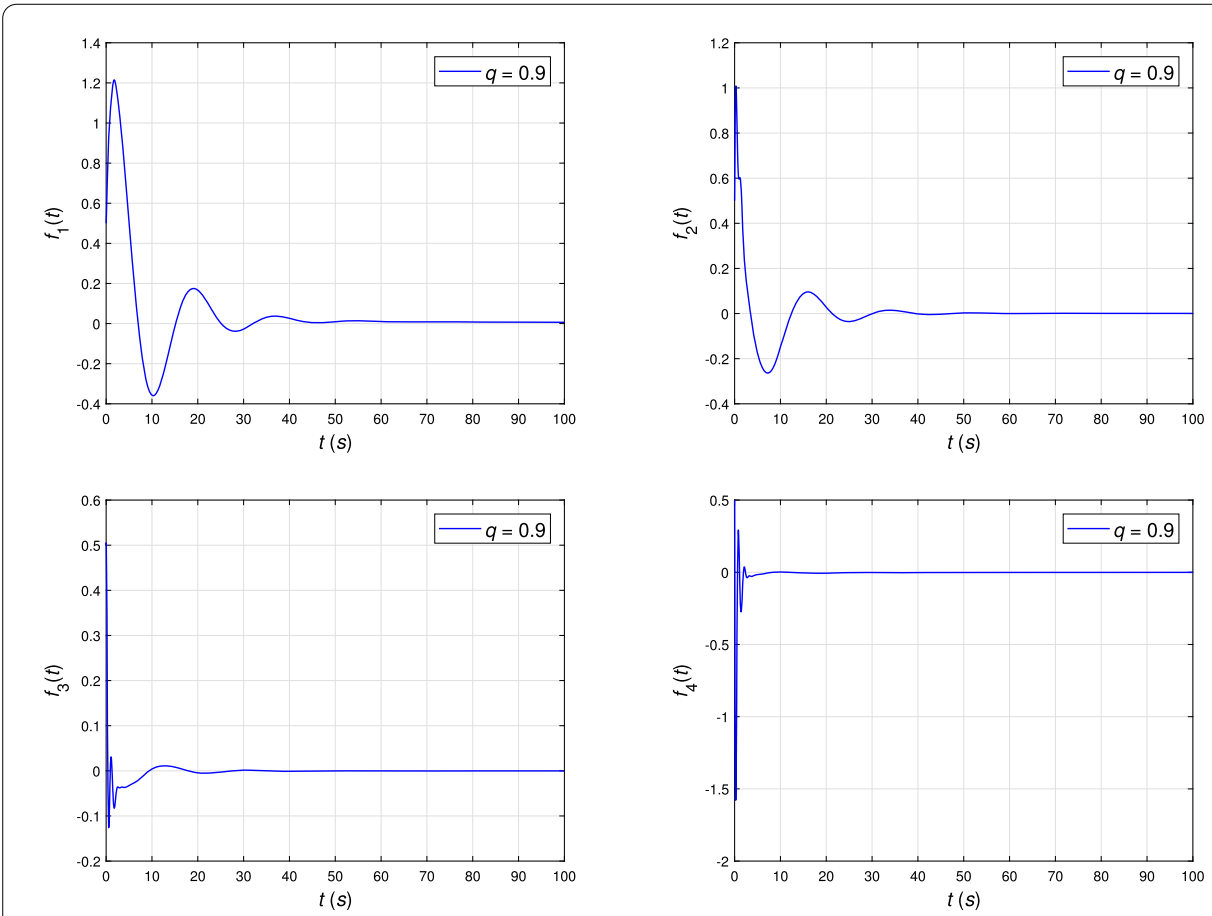

Figure 7 The time-domain responses of the controlled system (14) for $q=0.9$
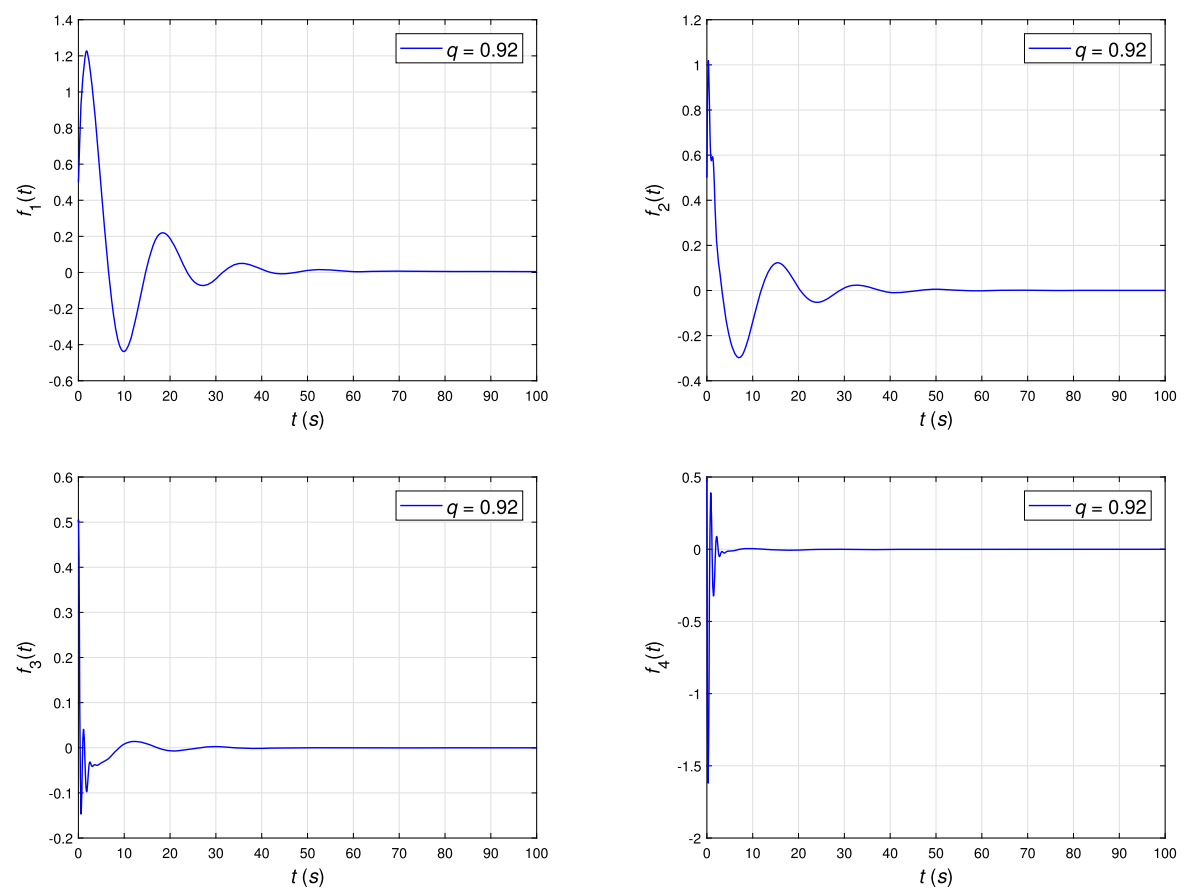

Figure 8 The time-domain responses of the controlled system (14) for $q=0.92$ 

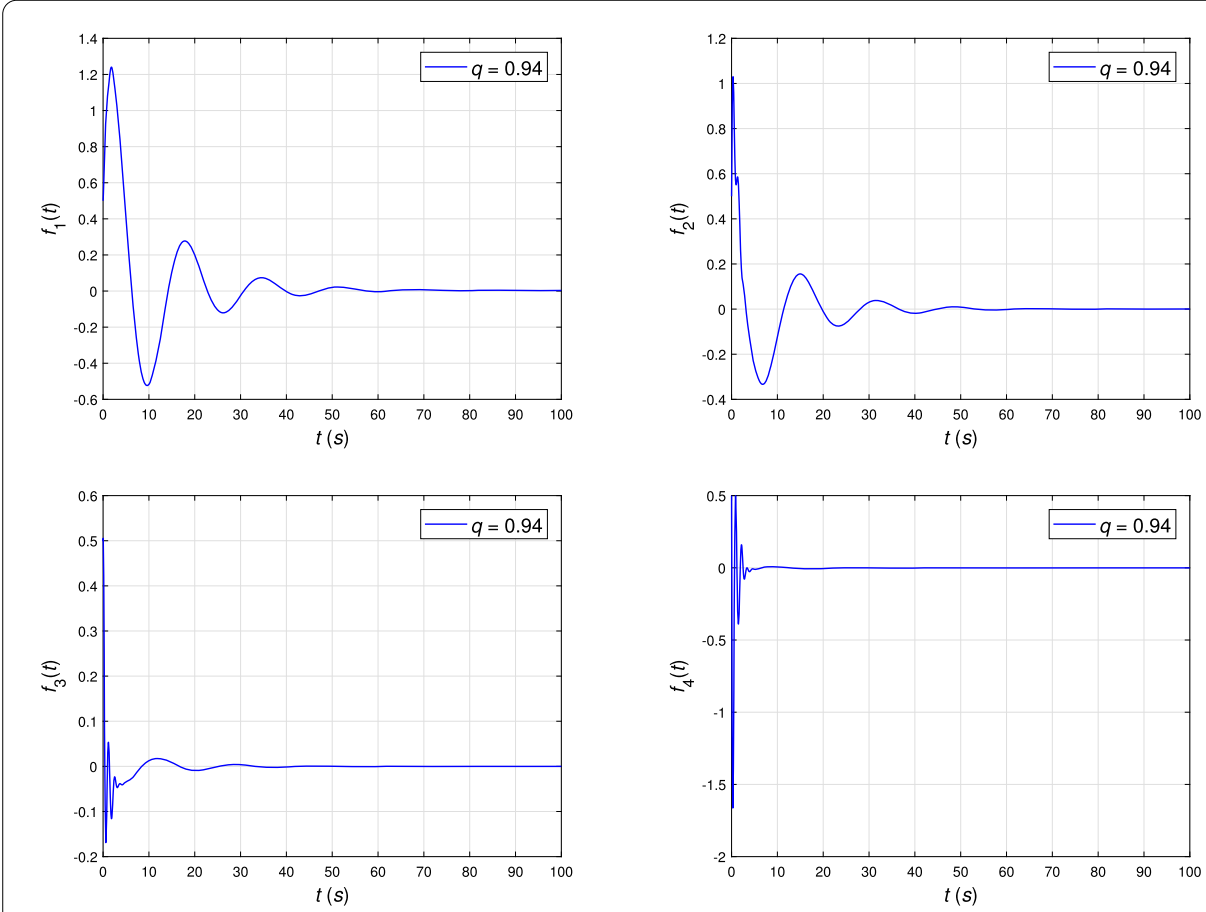

Figure 9 The time-domain responses of the controlled system (14) for $q=0.94$
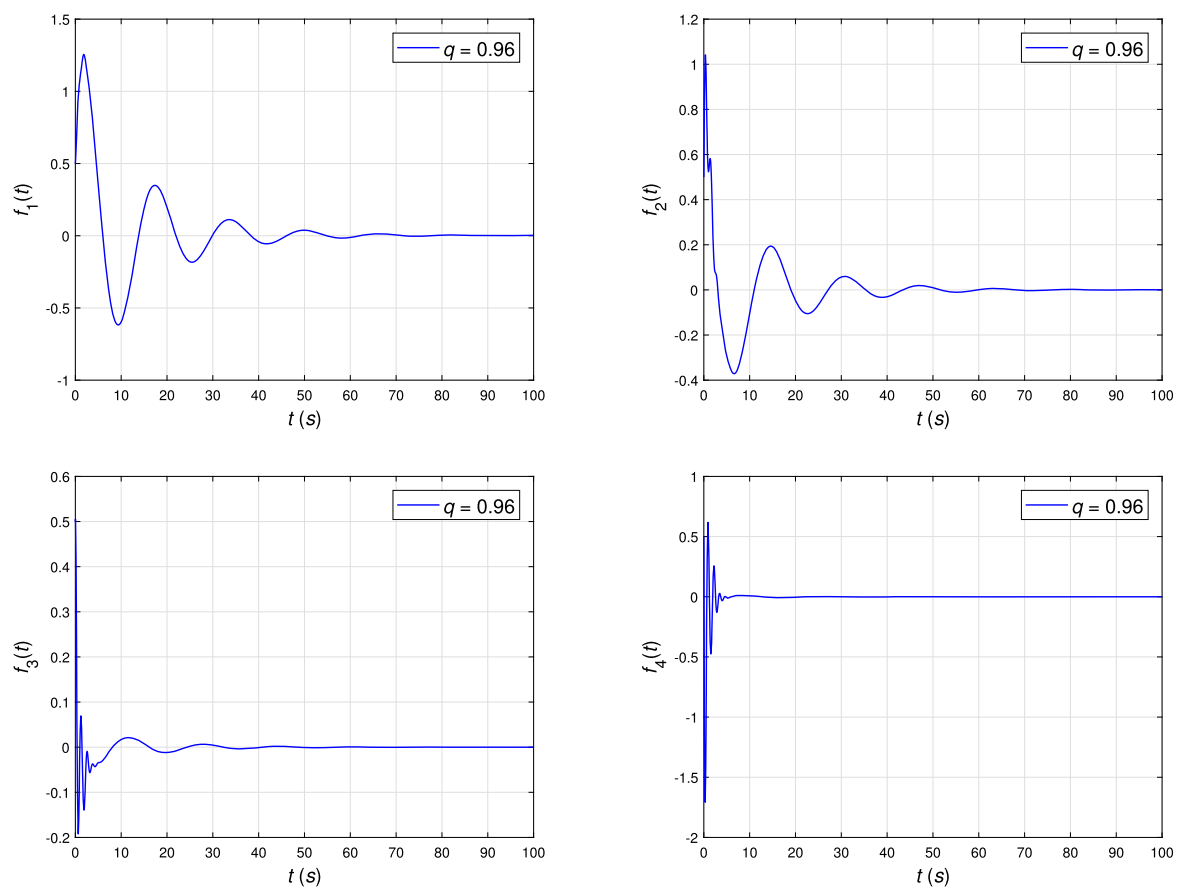

Figure 10 The time-domain responses of the controlled system (14) for $q=0.96$ 

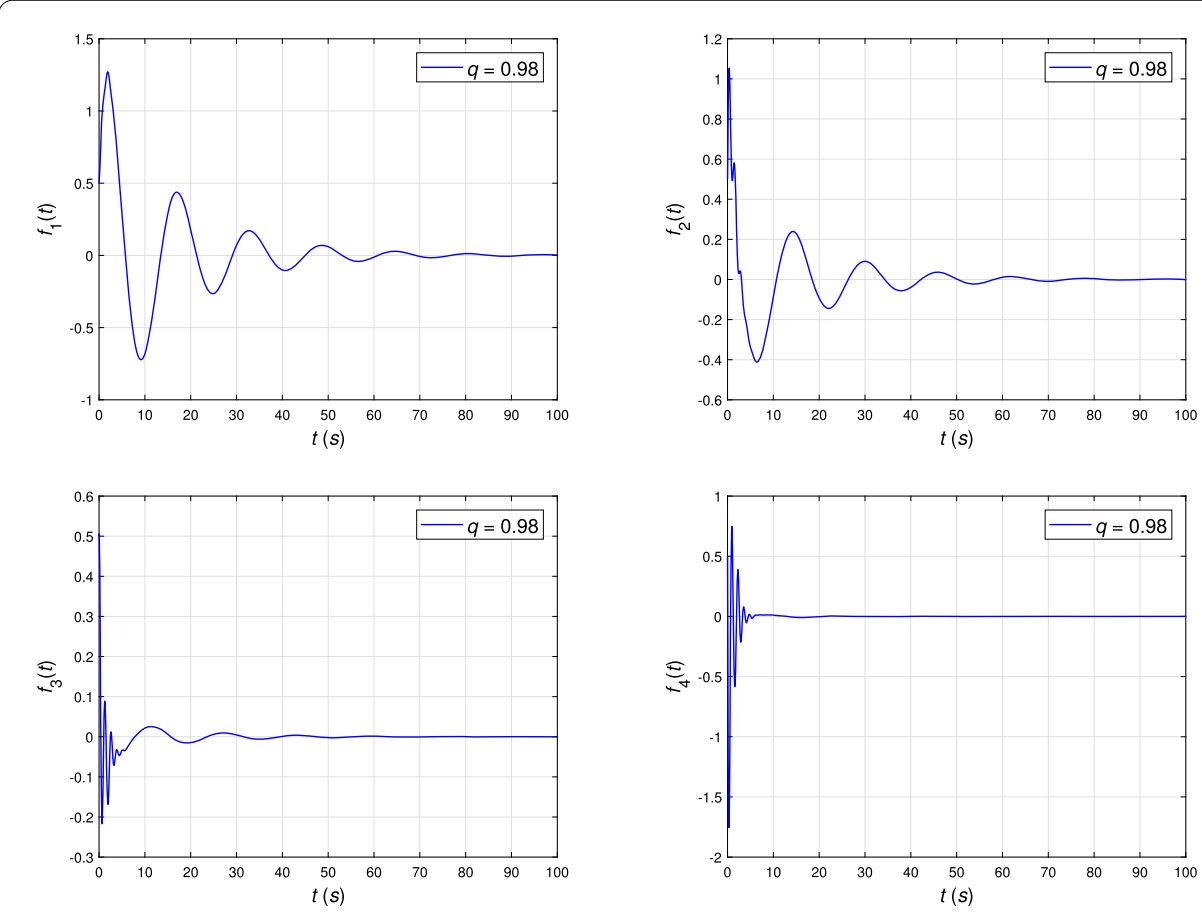

Figure 11 The time-domain responses of the controlled system (14) for $q=0.98$
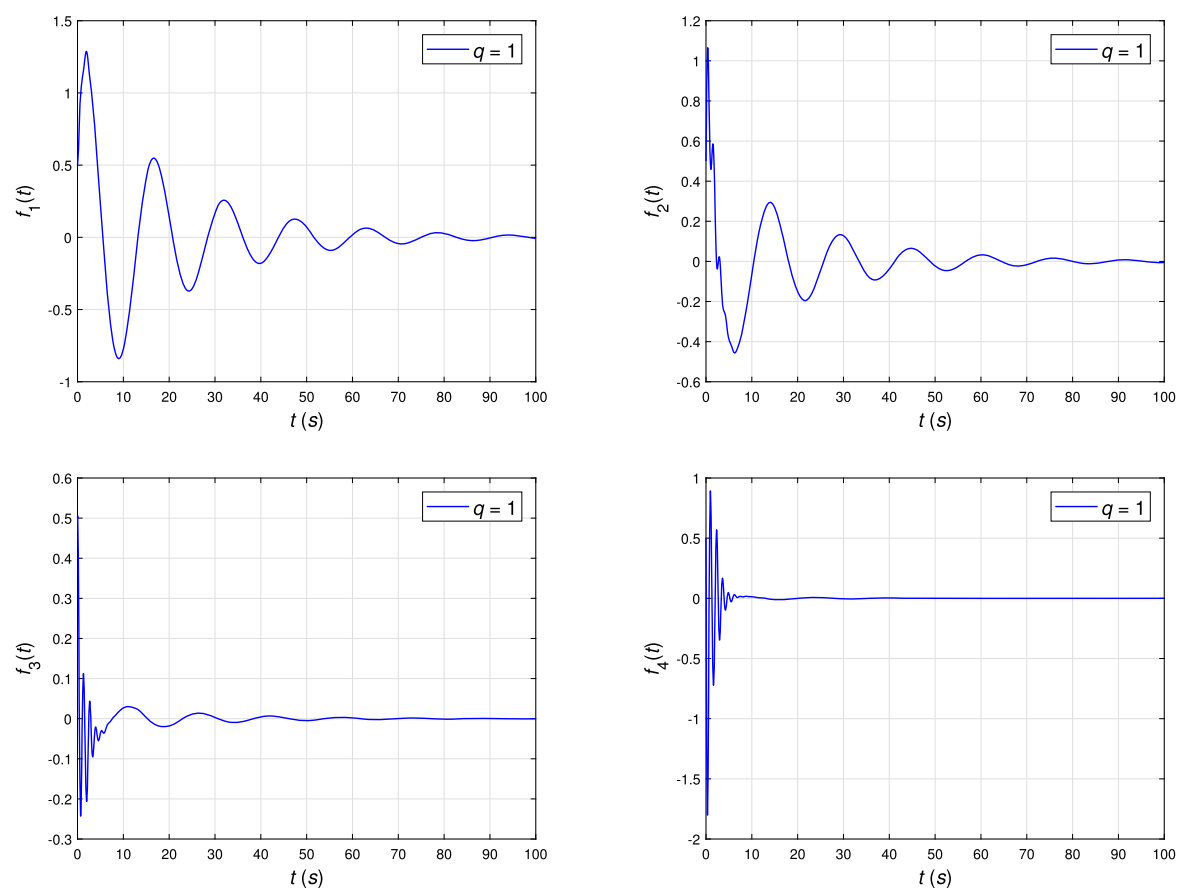

Figure 12 The time-domain responses of the controlled system (14) for $q=1$ 


$$
\left\{\begin{array}{l}
\frac{1}{{ }^{1-q}} C \mathscr{D}^{q} g_{1}=g_{2}+u_{1}, \\
\frac{1}{{ }^{1-q}} C \mathscr{D}^{q} g_{2}=\alpha g_{3}+u_{2}, \\
\frac{1}{{ }^{1-q}} C \mathscr{D}^{q} g_{3}=g_{4}+u_{3}, \\
\frac{1}{\theta^{1-q}} C \mathscr{D}^{q} g_{4}=-g_{1}-\beta g_{3}+\gamma g_{2}-g_{4}+\varepsilon g_{2}\left(1-g_{1}^{2}+\eta g_{1}^{4}-\kappa g_{1}^{6}\right)+u_{4},
\end{array}\right.
$$

where $f_{i}$ and $g_{i}$ are the states of the master and the slave systems, respectively, and $u_{i}$ is the control input. The objective is to design $u_{i}$ in such a way that the synchronization error goes to zero asymptotically. To reach this aim, we define the error of synchronization by $e_{i}(t)=g_{i}(t)-f_{i}(t)$. Then the error dynamics will be

$$
\left\{\begin{array}{l}
\frac{1}{{ }^{1-q} C} \mathscr{D}^{q} e_{1}=e_{2}+u_{1}, \\
\frac{1}{\theta^{1-q}} C \mathscr{D}^{q} e_{1} e_{2}=\alpha e_{3}+u_{2}, \\
\frac{1}{\bar{\theta}^{1-q}} C \mathscr{D}^{q} e_{1}=e_{4}+u_{3}, \\
{ }_{0}^{C} D_{t}^{\rho_{4}} e_{4}=-e_{1}-\beta e_{3}+(\gamma+\varepsilon) e_{2}-e_{4}-\varepsilon g_{2} g_{1}^{2}+\varepsilon f_{2} f_{1}^{2}+\varepsilon \eta g_{2} g_{1}^{4} \\
\quad-\varepsilon \eta f_{2} f_{1}^{4}-\varepsilon \kappa g_{2} g_{1}^{6}+\varepsilon \kappa f_{2} f_{1}^{6}+u_{4} .
\end{array}\right.
$$

Considering the control variables as

$$
\left\{\begin{array}{l}
u_{1}=v_{1}, \\
u_{2}=v_{2}, \\
u_{3}=v_{3}, \\
u_{4}=\varepsilon g_{2} g_{1}^{2}-\varepsilon f_{2} f_{1}^{2}-\varepsilon \eta g_{2} g_{1}^{4}+\varepsilon \eta f_{2} f_{1}^{4}+\varepsilon \kappa g_{2} g_{1}^{6}-\varepsilon \kappa f_{2} f_{1}^{6}+v_{4},
\end{array}\right.
$$

one attains

$$
\left\{\begin{array}{l}
\frac{1}{\theta^{1-q}} C \mathscr{D}^{q} e_{1}=e_{2}+v_{1}, \\
\frac{1}{{ }^{1-q}} C \mathscr{D}^{q} e_{1} e_{2}=\alpha e_{3}+v_{2}, \\
\frac{1}{\bar{\theta}^{1-q}} C \mathscr{D}^{q} e_{1}=e_{4}+v_{3}, \\
{ }_{0}^{C} D_{t}^{\rho_{4}} e_{4}=-e_{1}-\beta e_{3}+(\gamma+\varepsilon) e_{2}-e_{4}+v_{4},
\end{array}\right.
$$

where $v_{i}$ represents the active control input. Now, we select

$$
\left[\begin{array}{l}
v_{1} \\
v_{2} \\
v_{3} \\
v_{4}
\end{array}\right]=\mathbf{A}\left[\begin{array}{c}
e_{1} \\
e_{2} \\
e_{3} \\
e_{4}
\end{array}\right]
$$

where $\mathbf{A}$ is a constant matrix and selected in order to satisfy the stability condition in Lemma 3.1 for the error dynamics (23). Note that different choices exist for A; an appropriate one can be

$$
\mathbf{A}=\left[\begin{array}{cccc}
-1 & -1 & 0 & 0 \\
0 & -1 & -\alpha & 0 \\
0 & 0 & -1 & -1 \\
1 & -(\gamma+\varepsilon) & \beta & 0
\end{array}\right]
$$



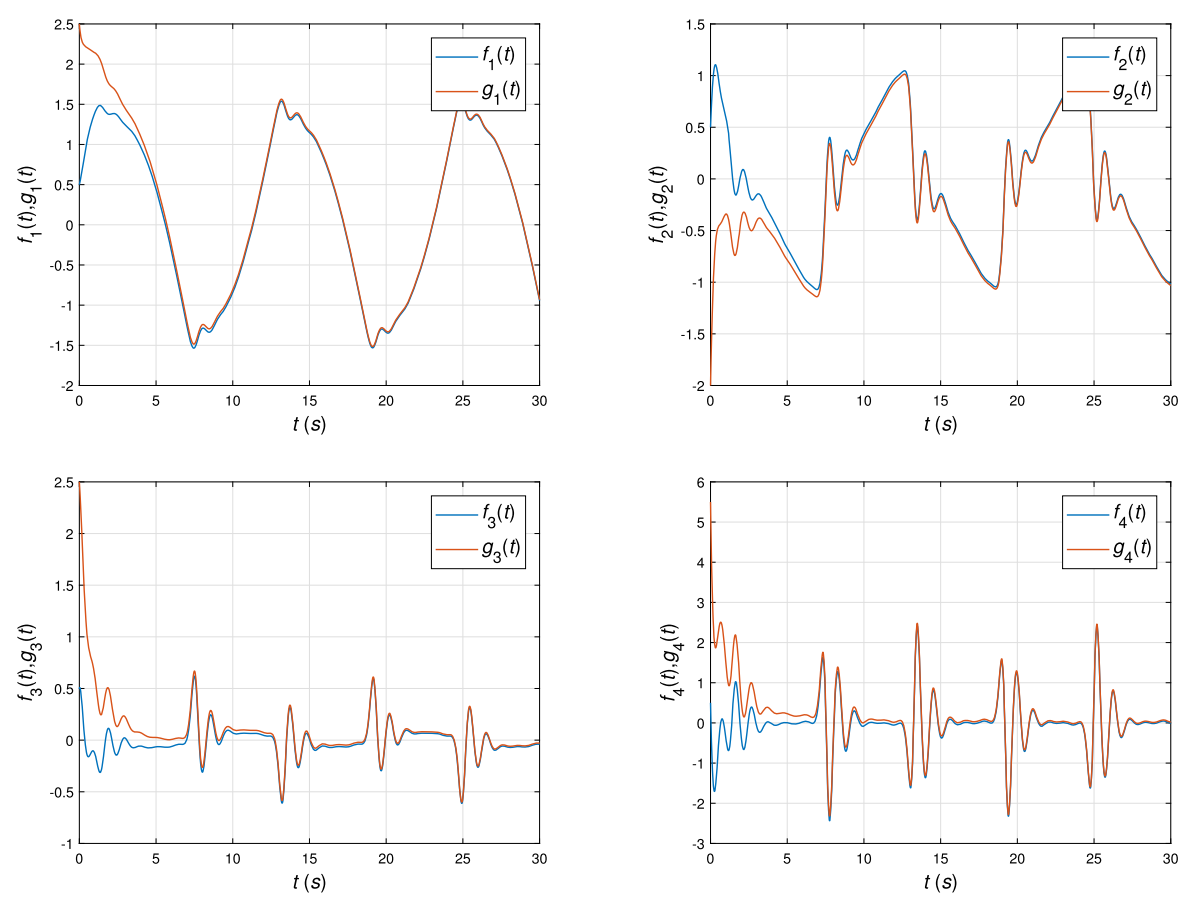

Figure 13 The state trajectories of the synchronized systems (19) and (20) for $q=0.9$
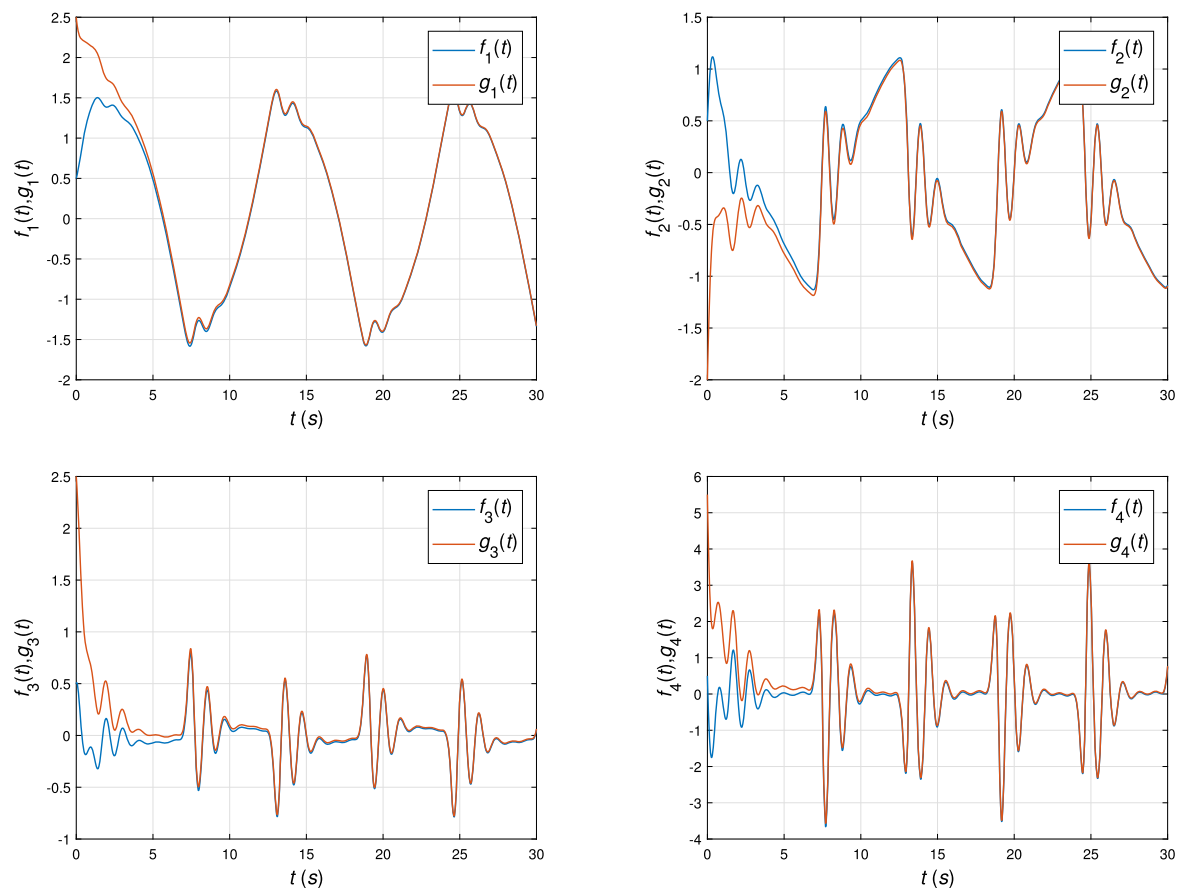

Figure 14 The state trajectories of the synchronized systems (19) and (20) for $q=0.92$ 

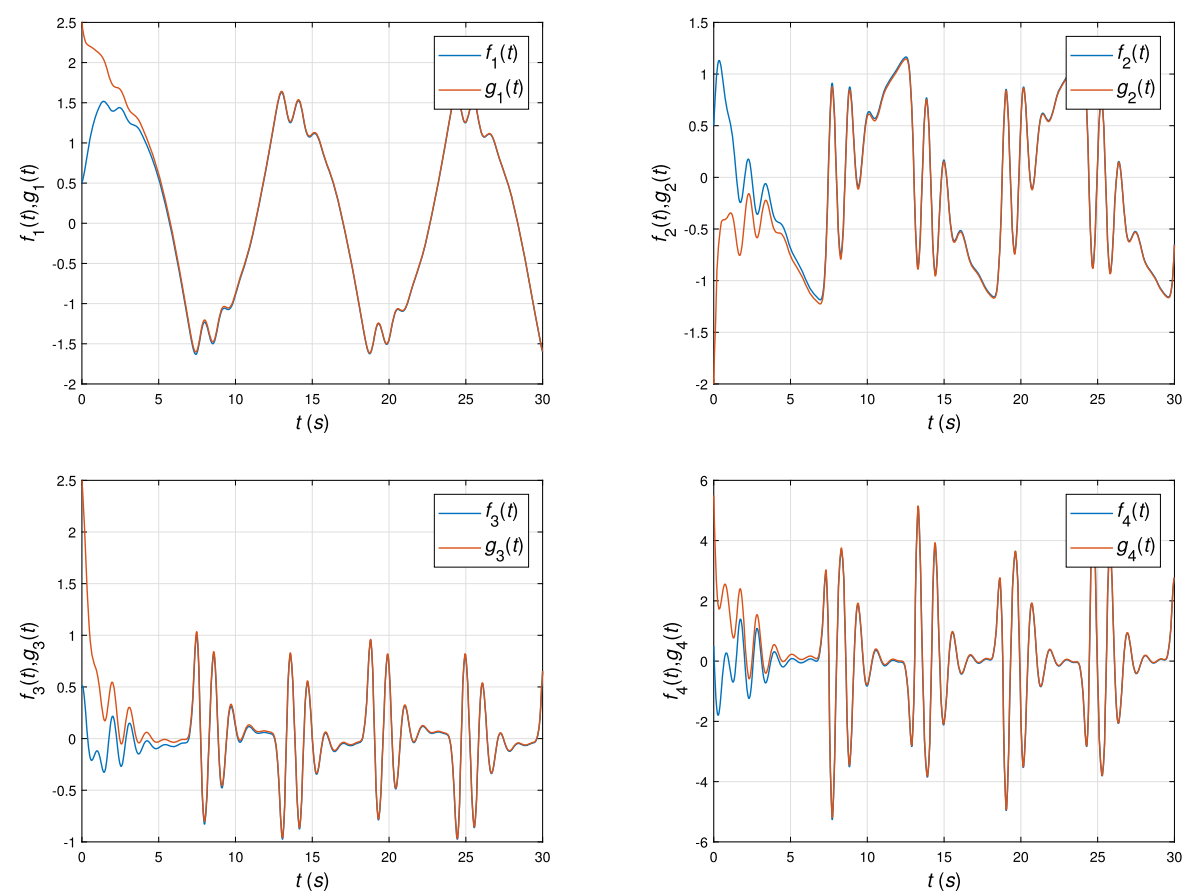

Figure 15 The state trajectories of the synchronized systems (19) and (20) for $q=0.94$
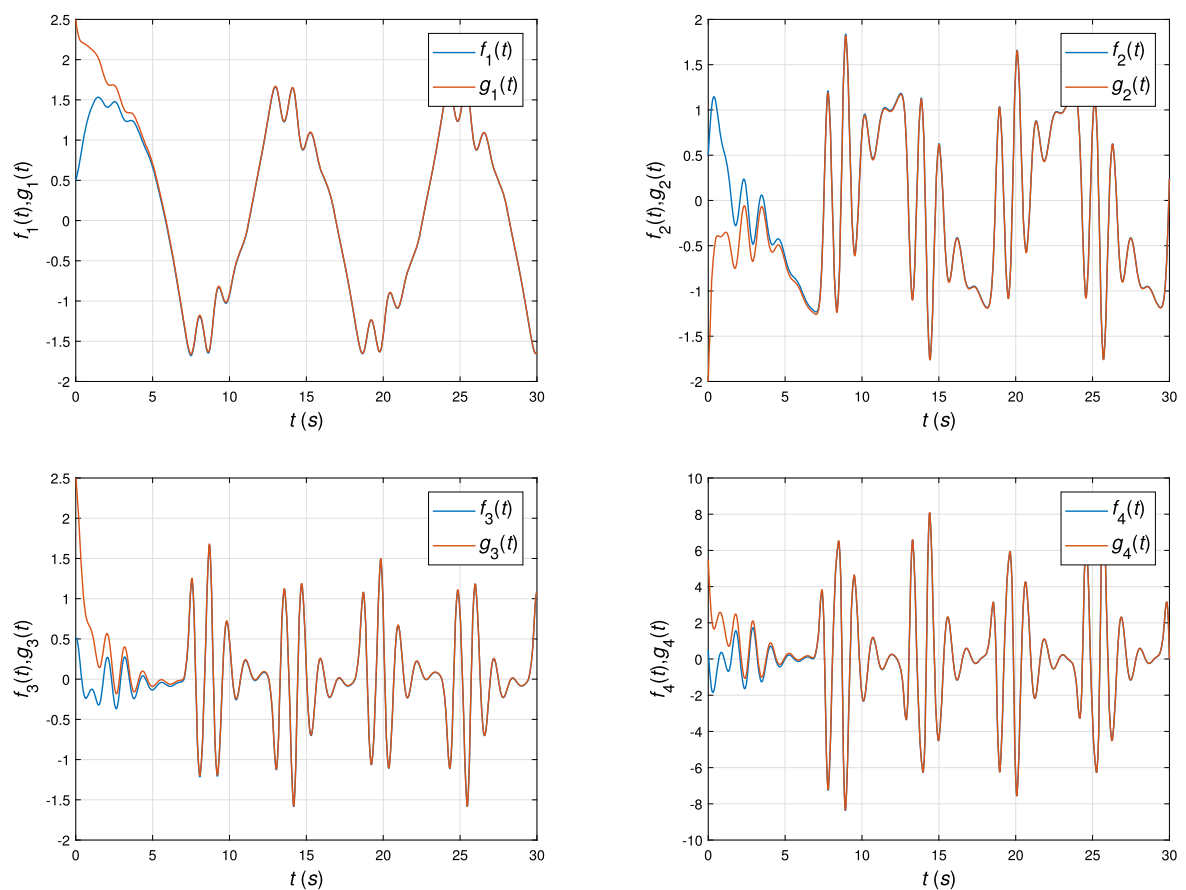

Figure 16 The state trajectories of the synchronized systems (19) and (20) for $q=0.96$ 

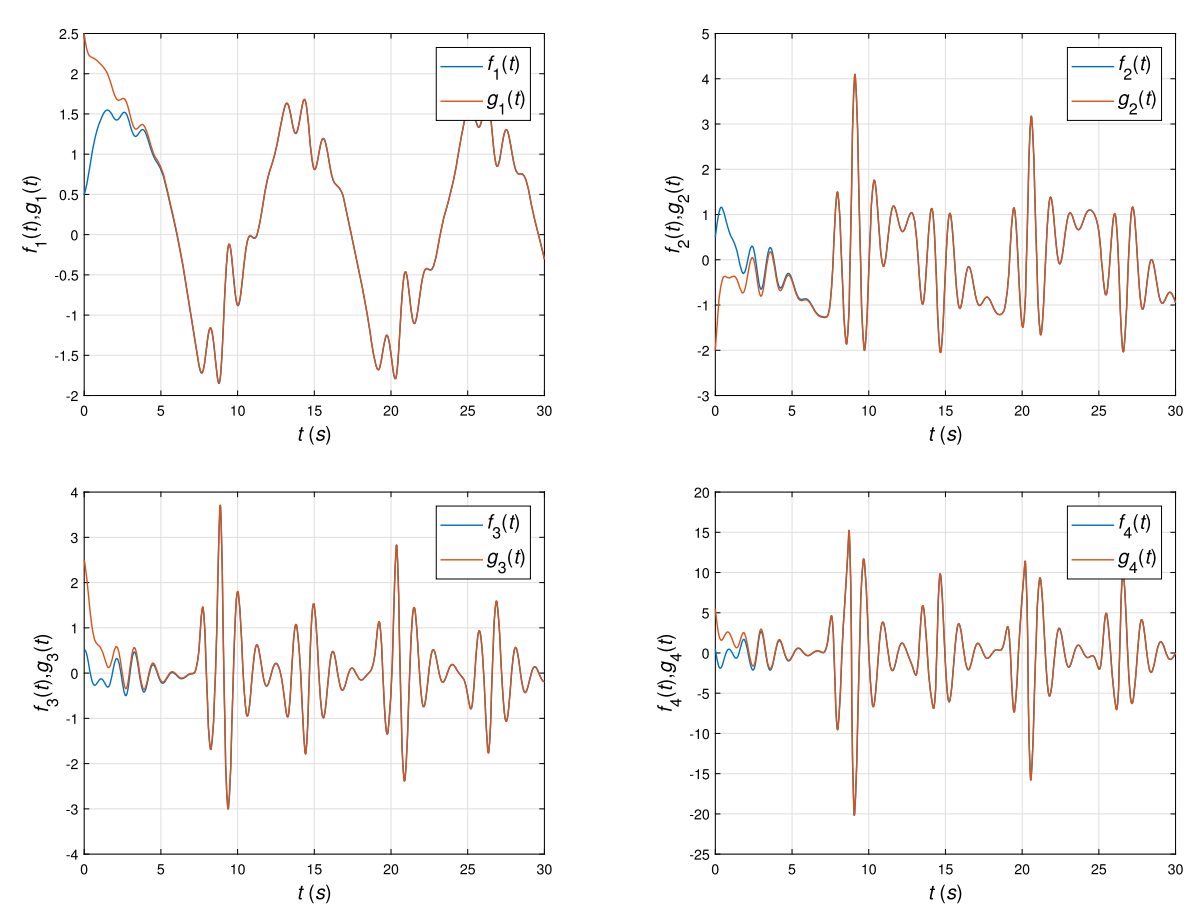

Figure 17 The state trajectories of the synchronized systems (19) and (20) for $q=0.98$
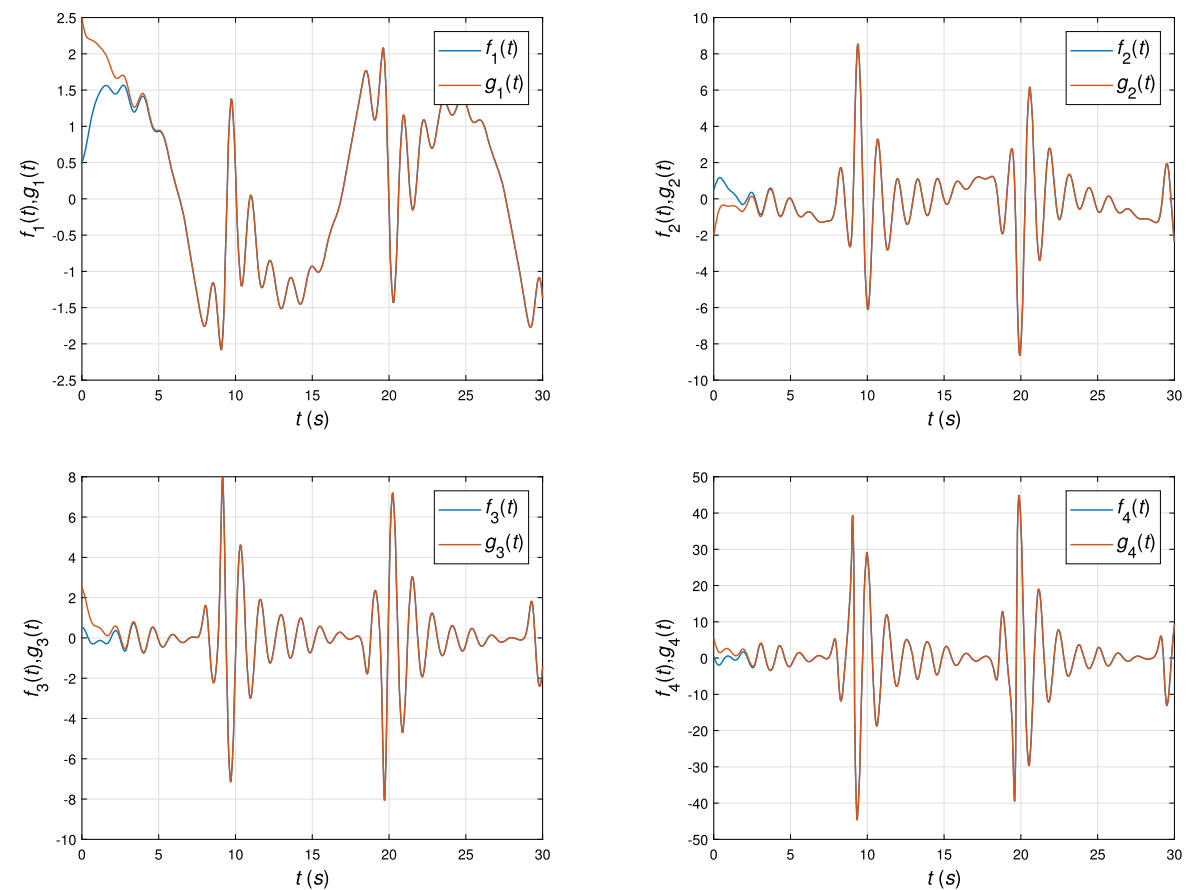

Figure 18 The state trajectories of the synchronized systems (19) and (20) for $q=1$ 
In addition, synchronization speed can be controlled by changing the main diagonal of matrix A.

Remark 4.1 Synchronization control can be regarded as a special tracking problem. When the master system is fixed in advance, the future dynamics of the master system are known, which can be considered as preview information. In this case, the speed of synchronization can be improved by using the preview control technique discussed in the new study [35].

To verify the theoretical synchronization approach mentioned above, we consider the initial states as

$$
\left\{\begin{array}{l}
f(0)=(0.5,0.5,0.5,0.5), \\
g(0)=(2.5,-2,2.5,5.5),
\end{array}\right.
$$

when the parameters and the fractional orders are $(\alpha, \beta, \gamma, \varepsilon, \eta, \kappa, \theta)=(5,24,-0.05,2.001$, $2.55,1.7,0.99)$ and $q=0.9,0.92,0.94,0.96,0.98,1$, respectively. Figures $13-18$ display the synchronized state variables verifying the proposed active control scheme.

\section{Conclusions}

The objective of this paper was to investigate the chaotic behaviour of a fractional biological system and compensate this undesirable behaviour by an efficient control strategy. In the first step, we discussed and explored the stability and the equilibrium of the considered model. Then we sketched the associated chaotic attractors in Figs. 1-6. Next, we designed a stabilizing state-feedback controller to overcome the undesirable chaotic behaviour. Afterwards, we introduced an active control design to synchronize two identical fractional biological oscillators. The validity of the chaos control and the synchronization approach was verified theoretically, and this analysis was also confirmed by some simulations in Figs. 7-18. Finally, as the future direction of this manuscript, we will consider more practical models, which will also be more complicated from the mathematical point of view.

\section{Acknowledgements}

This project was funded by the research and development office (RDO) at the ministry of Education, Kingdom of Saudi Arabia. Grant no. (HIQI-16-2019). The authors also, acknowledge with thanks research and development office (RDO-KAU) at King Abdulaziz University for technical support.

Funding

Research and development office (RDO) at the ministry of Education, Kingdom of Saudi Arabia. Grant no. (HIQI-16-2019).

Availability of data and materials

Data sharing is not applicable to this article as no datasets were generated or analysed during the current study.

Competing interests

The authors declare that they have no competing interests.

Authors' contributions

The authors declare that the study was realized in collaboration with equal responsibility. All authors read and approved the final manuscript.

\section{Author details}

Mathematical Modelling and Applied Computation Research Group (MMAC), Department of Mathematics, Faculty of Science, King Abdulaziz University, Jeddah 21589, Saudi Arabia. ${ }^{2}$ Department of Mathematics, Faculty of Arts and Sciences, Çankaya University, 06530 Ankara, Turkey. ${ }^{3}$ Institute of Space Sciences, P.O. Box, MG-23, R 76900,

Magurele-Bucharest, Romania. 


\section{Publisher's Note}

Springer Nature remains neutral with regard to jurisdictional claims in published maps and institutional affiliations.

\section{Received: 5 October 2020 Accepted: 7 January 2021 Published online: 21 January 2021}

\section{References}

1. Jajarmi, A., Hajipour, M., Baleanu, D.: New aspects of the adaptive synchronization and hyperchaos suppression of a financial model. Chaos Solitons Fractals 99, 285-296 (2017)

2. Sajjadi, S.S., Baleanu, D., Jajarmi, A., Mohammadi Pirouz, H.: A new adaptive synchronization and hyperchaos control of a biological snap oscillator. Chaos Solitons Fractals 138, 109919 (2020)

3. Grassi, G., Mascolo, S.: Nonlinear observer design to synchronize hyperchaotic systems via a scalar signal. IEEE Trans. Circuits Syst. I 44(10), 1011-1014 (1997)

4. Raikov, M., Balthazar, J.M.: On control and synchronization in chaotic and hyperchaotic systems via linear feedback control. Commun. Nonlinear Sci. Numer. Simul. 13(7), 1246-1255 (2008)

5. Al-Azzawi, S.F., Aziz, M.M.: Chaos synchronization of nonlinear dynamical systems via a novel analytical approach. Alex. Eng. J. 57(4), 3493-3500 (2018)

6. Chen, Z., Yuan, X., Yuan, Y., IU, H.H.-C., Fernando, T.: Parameter identification of chaotic and hyperchaotic systems using synchronization-based parameter observer. IEEE Trans. Circuits Syst. I 63(9), 1464-1475 (2016)

7. Batmani, Y.: Chaos control and chaos synchronization using the state-dependent Riccati equation techniques. Trans. Inst. Meas. Control 41(2), 311-320 (2019)

8. Al-Khedhairi, A., Matouk, A., Askar, S.: Computations of synchronisation conditions in some fractional order chaotic and hyperchaotic systems. Pramana 92(5), 72 (2019)

9. Othman, A.A., Noorani, M., Al-Sawalha, M.M.: Adaptive dual synchronization of chaotic and hyperchaotic systems with fully uncertain parameters. Optik 127(19), 7852-7864 (2016)

10. Azar, A.T., Serranot, F.E., Vaidyanathan, S.: Chapter 10 - Sliding mode stabilization and synchronization of fractional order complex chaotic and hyperchaotic systems. In: Mathematical Techniques of Fractional Order Systems: Advances in Nonlinear Dynamics and Chaos, pp. 283-317 (2018)

11. Dongmo, E.D., Ojo, K.S., Woafo, P., Njah, A.N.: Difference synchronization of identical and nonidentical chaotic and hyperchaotic systems of different orders using active backstepping design. J. Comput. Nonlinear Dyn. 13(5), 051005 (2018)

12. Sabarathinam, S., Thamilmaran, K.: Controlling of chaos in a tumour growth cancer model: an experimental study. Electron. Lett. 54(20), 1160-1162 (2018)

13. El-Gohary, A.: Chaos and optimal control of equilibrium states of tumor system with drug. Chaos Solitons Fractals $41(1), 425-435(2009)$

14. Vaidyanathan, S.: Global chaos synchronization of the Lotka-Volterra biological systems with four competitive species via active control. Int. J. PharmTech Res. 8(6), 206-217 (2015)

15. Wu, G.C., Baleanu, D., Xie, H.P., Chen, F.L.: Chaos synchronization of fractional chaotic maps based on the stability condition. Physica A 460, 374-383 (2016)

16. Boubellouta, A., Zouari, F., Boulkroune, A.: Intelligent fuzzy controller for chaos synchronization of uncertain fractional-order chaotic systems with input nonlinearities. Int. J. Gen. Syst. 48(3), 211-234 (2019)

17. Asad, J.H., Baleanu, D., Ghanbari, B., Jajarmi, A., Mohammadi Pirouz, H.: Planar system-masses in an equilateral triangle: numerical study within fractional calculus. Comput. Model. Eng. Sci. 124(3), 953-968 (2020)

18. Baleanu, D., Jajarmi, A., Sajjadi, S.S., Asad, J.H.: The fractional features of a harmonic oscillator with position-dependent mass. Commun. Theor. Phys. 72(5), 055002 (2020)

19. Jajarmi, A., Yusuf, A., Baleanu, D., Inc, M.: A new fractional HRSV model and its optimal control: a non-singular operator approach. Physica A 547, 123860 (2020)

20. Baleanu, D., Jajarmi, A., Mohammadi, H., Rezapour, S.: A new study on the mathematical modelling of human liver with Caputo-Fabrizio fractional derivative. Chaos Solitons Fractals 134, 109705 (2020)

21. Baleanu, D., Jajarmi, A., Sajjadi, S.S., Asad, J.H.: A new feature of the fractional Euler-Lagrange equations for a coupled oscillator using a nonsingular operator approach. Front. Phys. 7, 196 (2019)

22. Baleanu, D., Sajjadi, S.S., Jajarmi, A., Defterli, O., Asad, J.H.: The fractional dynamics of a linear triatomic molecule. Rom. Rep. Phys. 73(1), 105 (2021)

23. Rezapour, S., Mohammadi, H., Jajarmi, A.: A new mathematical model for Zika virus transmission. Adv. Differ. Equ. $2020,589(2020)$

24. Jajarmi, A., Baleanu, D.: A new iterative method for the numerical solution of high-order nonlinear fractional boundary value problems. Front. Phys. 8, 220 (2020)

25. Jajarmi, A., Baleanu, D.: On the fractional optimal control problems with a general derivative operator. Asian J. Control (2019). https://doi.org/10.1002/asjc.2282

26. Mohammadi, F., Moradi, L., Baleanu, D., Jajarmi, A.: A hybrid functions numerical scheme for fractional optimal contro problems: application to non-analytic dynamical systems. J. Vib. Control 24(21), 5030-5043 (2018)

27. Kilbas, A.A., Srivastava, H.H., Trujillo, J.J.: Theory and Applications of Fractional Differential Equations. Elsevier, New York (2006)

28. Vaidyanathan, S., Feki, M., Sambas, A., Lien, C.H.: A new biological snap oscillator: its modelling, analysis, simulations and circuit design. Int. J. Simul. Process Model. 13(5), 419-432 (2018)

29. Gómez-Aguilar, J.F., Rosales-García, J.J., Bernal-Alvarado, J.J., Córdova-Fraga, T., Guzmán-Cabrera, R.: Fractional mechanical oscillators. Rev. Mex. Fis. 58, 348-352 (2012)

30. Matouk, A.E.: Stability conditions, hyperchaos and control in a novel fractional order hyperchaotic system. Phys. Lett. A 373(25), 2166-2173 (2009)

31. Dorf, R.C., Robert, H.B.: Modern Control Systems. Prentice Hall, New York (2001)

32. Diethelm, K., Freed, A.D.: The FracPECE Subroutine for the Numerical Solution of Differential Equations of Fractional Order. Forschungund wissenschaftliches Rechnen (1998); Heinzel, S., Plesser, T., Eds. Gesellschaft für wissenschaftliche Datenverarbeitung. Göttingen, Germany (1999) 
33. Hairer, E., Lubichm, C., Schlichte, M.: Fast numerical solution of nonlinear Volterra convolution equations. SIAM J. Sci. Stat. Comput. 6(3), 532-541 (1985)

34. Diethelm, K., Ford, N.J., Freed, A.D.: Detailed error analysis for a fractional Adams method. Numer. Algorithms 36(1), 31-52 (2004)

35. Yu, X., Li, L.: Trajectory tracking control with preview action for a class of continuous-time Lur'e-type nonlinear systems. Adv. Differ. Equ. 2020, 293 (2020)

Submit your manuscript to a SpringerOpen ${ }^{\circ}$ journal and benefit from:

- Convenient online submission

- Rigorous peer review

- Open access: articles freely available online

- High visibility within the field

- Retaining the copyright to your article

Submit your next manuscript at $\gg$ springeropen.com 